\title{
Effect of Different Post-treatments on the Microstructure of EBM-Built Alloy 718
}

\author{
Sneha Goel (D), Magnus Ahlfors, Fouzi Bahbou, and Shrikant Joshi
}

(Submitted March 23, 2018; in revised form August 30, 2018; published online October 29, 2018)

\begin{abstract}
Electron beam melting (EBM) of Alloy 718 is of rapidly growing interest as it allows cost-effective production of complex components. However, the inherent flaws in the component in as-built state are of concern in view of the severe working conditions in which Alloy 718 components typically operate. The present work entails an investigation of changes in microstructure that accompany some post-treatments that are being widely considered to address defects in EBM processed Alloy 718 . The effect of two different post-treatments, namely hot isostatic pressing (HIP) and a combined HIP + heat treatment (HT) carried out inside the HIP vessel, have been studied and results from as-built and post-treated specimens were compared in terms of porosity/lack-of-fusion, microstructure, phase constitution (NbC content, $\delta$-phase) and micro-hardness. Post-treatment resulted in reduction in defect content by more than an order of magnitude. HIPing led to complete dissolution of $\delta$ phase. In comparison to as-built material, HIPed specimens exhibited significant drop in hardness. However, a sharp 'recovery' of hardness to yield values higher than in as-built condition was observed after HIP + HT and can be attributed to precipitation of $\gamma^{\prime \prime}$ phase.
\end{abstract}

Keywords additive manufacturing, alloy 718 , electron beam melting, hardness, heat treatment, HIP, microstructure

\section{Introduction}

Additive manufacturing (AM) is the process of fabrication of parts through layer-by-layer addition of material. This allows manufacture of extremely complex parts intended for structural and functional applications, such as turbine blades with unique cooling channels. There is a growing interest in production through AM of components from high temperature materials like Alloy 718, a Ni-Fe based superalloy widely used in the aerospace industry. Although Alloy 718 (also known as Inconel 718 ) in wrought and cast form has been widely studied, there is a need for a detailed analysis of AM built material as the latter exhibits different microstructures and properties in comparison to those manufactured through the conventional manufacturing routes. AM processes commonly used for fabrication of Alloy 718 components are directed metal deposition (DMD), which uses a laser source with wire or powder as the feedstock, and powder-bed fusion techniques such as selective laser melting (SLM) and electron beam melting (EBM). This study examined EBM fabrication of Alloy 718.

This article is an invited paper selected from presentations at the symposium "Additive Manufacturing of Metals: Post Processing," held during MS\&T'17, October 8-12, 2017, in Pittsburgh, Pa., and has been expanded from the original presentation.

Sneha Goel and Shrikant Joshi, University West, Department of Engineering Science, Trollhättan, Sweden; Magnus Ahlfors, Quintus Technologies AB, Västerås, Sweden; Fouzi Bahbou, ARCAM AB, Mölndal, Sweden. Contact e-mails: sneha.goel@hv.se, snehagoel27@gmail.com.
Thermal post-treatment of AM-builds is actively considered to enhance mechanical integrity. Hence, changes in microstructure during such treatments are also pertinent to monitor. Generally, thermal post-treatment involves hot isostatic pressing (HIP) for closure and healing of defects, solution treatment for homogenization of the microstructure, and aging for precipitation of strengthening phase $\left(\gamma^{\prime \prime}, \mathrm{Ni}_{3} \mathrm{Nb}\right.$ and $\gamma^{\prime}, \mathrm{Ni}_{3}(\mathrm{Al}$, Ti)). The specific interest in thermal post-treatment of EBMbuilt Alloy 718 is motivated by published results showing substantial improvement in mechanical performance after posttreatment (Ref 1-3).

The present study comprehensively investigates the influence of two different post-treatments, namely HIP and HIP + heat treatment (HT). The latter involved HIP, solution treatment, and aging carried out as a single cycle within the HIP vessel. The influence of post-treatment on grain structure, defect concentration, phase constitution, and micro-hardness have been investigated.

\section{Experiment}

\subsection{EBM Build}

The EBM build utilized for the present study was constructed at Arcam AB (Mölndal, Sweden). A plasma atomized powder (nominal size range: $45-106 \mu \mathrm{m}$ ) supplied by AP\&C (Montréal, Canada) was used. The chemical composition of the powder is given in Table 1. A high temperature steel base plate was preheated to $\sim 1000{ }^{\circ} \mathrm{C}$ before initiating the EBM building process. The deposition cycle for each layer consisted of 6 stages: (1) pre-heating of the powder layer being processed, (2) localized pre-heating of regions to be melted, (3) contour melting of the frame of the parts, (4) hatch melting of the interior of the parts, (5) post-heating of the layer being processed, and (6) lowering of powder bed and powder raking to form a uniform layer of $75 \mu \mathrm{m}$ for next cycle. An EBM 
Table 1 Chemical composition of the Alloy 718 powder used in the present study

\begin{tabular}{lccccccccc}
\hline Element, wt.\% & $\mathbf{N i}$ & $\mathbf{C r}$ & $\mathbf{F e}$ & $\mathbf{N b}+\mathbf{T a}$ & $\mathbf{M o}$ & $\mathbf{T i}$ & $\mathbf{A l}$ & $\mathbf{C}$ & $\mathbf{N}$ \\
\hline Powder alloy 718 & 51.67 & 19.09 & $\sim 19.33$ & 5.31 & 3.12 & 0.89 & 0.53 & 0.04 & 0.02 \\
\hline
\end{tabular}

Control software version 4.2.76 (ARCAM AB, Mölndal, Sweden) and an accelerating voltage of $60 \mathrm{kV}$ were used for production. Figure 1 shows the EBM build used in the present study and the rod structures $(100 \mathrm{~mm}$ length $\times 15 \mathrm{~mm}$ diameter) observed therein were the subject of the present study. The rods labelled 1-3 were extensively characterized both to ascertain microstructural uniformity within a single rod, as well as between rods. This was done before subjecting parts of different rod specimens to post-treatments described below, to ensure that differences noted during subsequent comparisons between as-built and post-treated specimens would be attributable to the post-treatment protocols alone.

\subsection{Post-treatment}

Two of the rods were subjected to two different post treatments, one involving HIP and the other including HIP + HT combined in a single cycle inside the HIP vessel. The time-temperature-pressure protocols associated with the two post-treatments studied, with the process graphs shown in Fig. 2, were: (a) HIP: $1200{ }^{\circ} \mathrm{C}$ for $4 \mathrm{~h}$ at $120 \mathrm{MPa}$, followed by rapid cooling to room temperature, and (b) HIP $+\mathrm{HT}$ : $1185^{\circ} \mathrm{C}$ for $3 \mathrm{~h}$ at $170 \mathrm{MPa}$, followed by furnace cooling to solution treatment temperature of $980{ }^{\circ} \mathrm{C}$ and holding for $1 \mathrm{~h}$, followed by rapid cooling to room temperature, thereafter $1 \mathrm{st}$ age hardening by heating to $740{ }^{\circ} \mathrm{C}$, holding for $8 \mathrm{~h}$, rapid cooling to $635^{\circ} \mathrm{C}$ for 2 nd age-hardening, holding for $10 \mathrm{~h}$ and followed by rapid cooling to room temperature. It may be noted that the HIP conditions in the above HIP and HIP + HT are slightly different since, given the high cost of conducting dedicated runs in an industrial large-volume HIP furnace, two already planned customer runs for additively manufactured Alloy 718 builds were utilized for this initial study. In this context, it is relevant to point out that, previously reported work on Alloy 718 has shown that change in time from $3 \mathrm{~h}$ to $4 \mathrm{~h}$ (Ref 4) and increase in pressure by $50 \mathrm{MPa}$ (Ref 5) resulted in only modest change in porosity content. Consequently, the temperature-time-pressure schedules utilized herein were deemed reasonable for a preliminary investigation.

\subsection{Characterization}

As mentioned elsewhere in this paper, microstructural uniformity within a built rod, as well as between rods located at different positions within the build, was first assessed using rods labelled 1, 2, and 3 in Fig. 1. For microstructure analysis to examine uniformity within a rod, $7-10 \mathrm{~mm}$ long sections along the build direction were taken from near the top, middle, and bottom regions of rod 2 using precision cutting. All the specimens were hot mounted and semi-automatically polished using Buehler PowerPro 5000 (Buehler, USA) system. The polished specimens were etched via electrolytic (2-4 V) etching using oxalic acid and examined under an optical microscope (OM) (Olympus BX60 M, HOFSTRAgroup ${ }^{\circledR}$, US) and scanning electron microscopes (SEMs) (HITACHI TM3000, Zeiss EVO 50). Image analysis was employed for quantification of

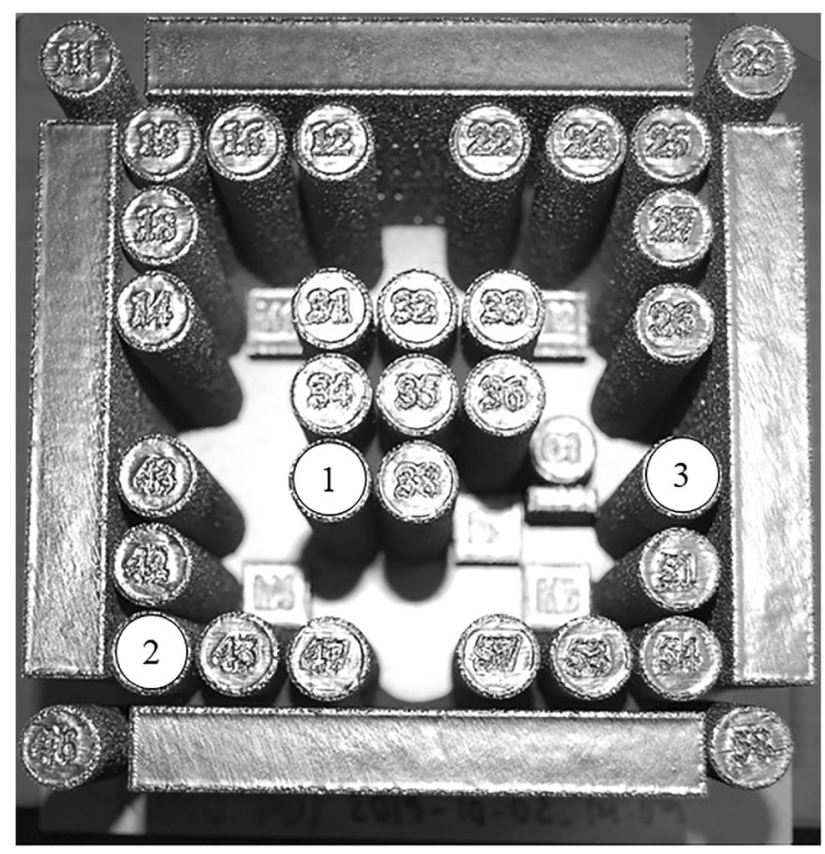

Fig. 1 Photograph of the EBM build. Rods from different positions within the build and labelled 1, 2, and 3 were investigated. Build direction is out of the plane of the paper

porosity and carbide content (using ASTM E1245-03 automatic image analysis method (Ref 6) and the open source ImageJ software) using at least 10 images in each case, and the average value \pm the confidence interval has been reported herein as recommended in the above ASTM standard. An energy dispersive spectroscopy (EDS) (Oxford instruments, UK) detector mounted on the Zeiss EVO 50 SEM was used for elemental analysis. Vickers micro-hardness testing (HMV-2, Shimadzu Corp., Japan) on the polished cross-sections was performed using $500 \mathrm{~g}$ load applied for $15 \mathrm{~s}$ in ambient conditions. For each of the specimens tested, 8-12 indents were recorded.

\section{Results and Discussion}

A detailed investigation of the role of post-treatment was preceded by a comprehensive study to assess microstructural uniformity not only within a rod but also among different rods constituting the build. This was crucial to ensure that the microstructural changes noticed during post-treatment were mainly attributable to the HIP and HIP + HT protocols used and not to any significant inherent differences in the different specimens used. It should be noted that, for characterization of a rod along the build direction, $5 \mathrm{~mm}$ of the very top and very bottom of the rod (representing regions near end-of-build and in immediate vicinity of the base plate, respectively) have been 

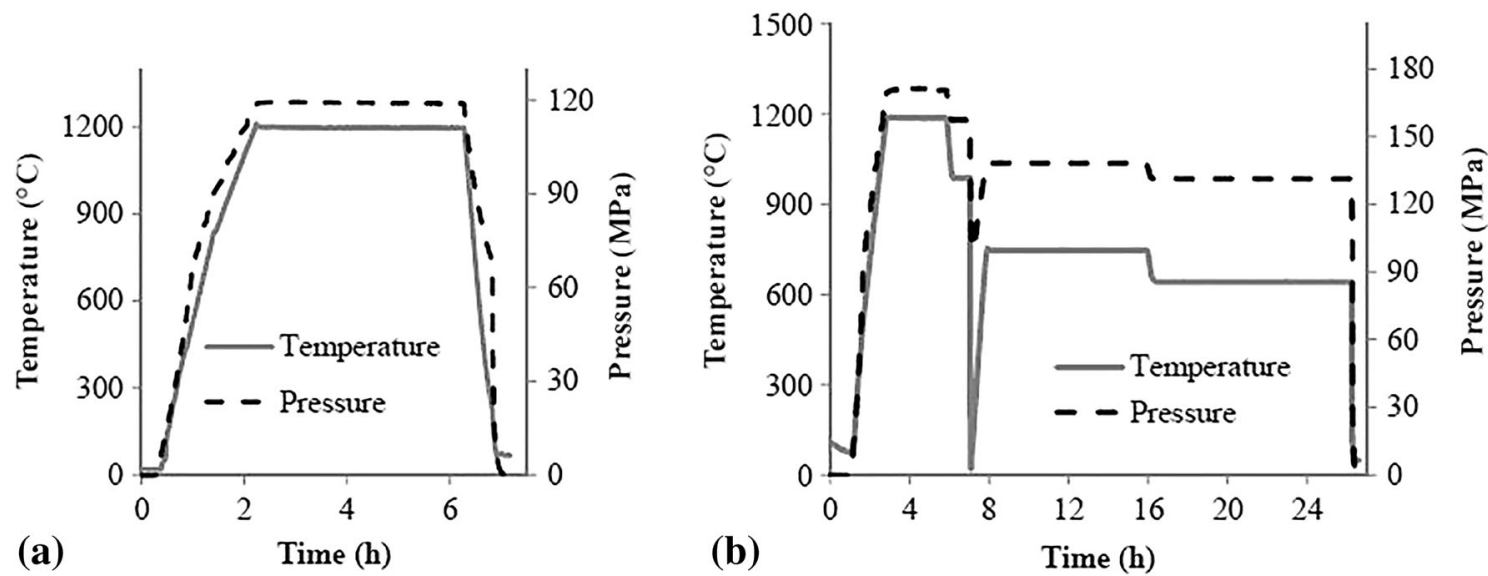

Fig. 2 Time-temperature-pressure graphs of (a) HIP and (b) HIP + HT post-treatments investigated

excluded for the limited scope of this study. At the beginning of part production the energy input is lowered, which could potentially lead to excessive defects in the very bottom region of the build. Previously published work has shown presence of Laves phase and microstructural gradients in the very top part of a build (Ref 7).

\subsection{Microstructure of As-Built Specimens}

For microstructure analysis along the build height, specimens near the top, middle and bottom of rod 2 were studied. In addition, uniformity in different rods within a build was assessed using specimens near the middle of the three different as-built rods (labelled 1, 2, and 3 in Fig. 1) along the build direction. The different microstructural features observed are discussed in the following sections.

3.1.1 Grain Structure. 3.1.1.1 Uniformity Within a Built Rod. The SEM (back-scattered electron, BSE) micrographs shown in Figs. 3 (a), (b), and (c) reveal the columnar grain structure oriented in the build direction of rod 2. Such directional solidification in the EBM process, resulting from the presence of large thermal gradients in the solidifying melt pool, has been widely reported (Ref 8,9$)$. Long grains up to an order of $1 \mathrm{~mm}$ were observed. As evident from Fig. 3(a), (b), and (c), no apparent difference in grain width was noticed along the build height of rod 2. Kirka et al. (Ref 7) have also reported such uniformity along the build height, except for the few $\mathrm{mm}$ portion at the top of the build. A previously reported study on EBM processed Ti-6Al-4 V had also found no significant variability in grain width with build height (Ref 10). It is pertinent to note that, in contrast to this observation, prior published work on SLM Alloy 718 builds have indicated higher columnar grain width near the top region of the component and lower grain width near the bottom region (Ref 11). The above variation in microstructure with build height in case of SLM was attributed to the difference in cooling rate, which is higher near the bottom, i.e., closer to the base plate, and lower near the top region. Hence, the observed uniformity in grain width along the build height in the present study is perhaps indicative of relatively uniform cooling rate with build height in the EBM builds investigated herein.

3.1.1.2 Uniformity in Different Rods Within a Build. SEM micrographs of the middle region of the as-built rods 1,2 , and 3 are shown in Fig. 3(d), (b), and (e), respectively. Comparison of the micrographs reveals similar columnar grain structure and grain width in the three as-built rods. This is suggestive of substantial rod-to-rod microstructural uniformity within the build, which needed to be confirmed before undertaking any comparative study by subjecting specimens from different rods to distinct post-treatments.

3.1.2 Defects. The defects observed in the as-built EBM Alloy 718 specimens can be categorized into the following categories: (a) gas porosity, (b) shrinkage porosity, and (c) lackof-fusion as shown in Fig. 4. As labelled in the figure, the gas pores appear circular in two dimensions, shrinkage pores are aligned with the build direction, and lack-of-fusion defects are perpendicular to the build direction. The reasons for occurrence of such defects have been explained previously (Ref 12). The total defect content in the specimens is summarized in Fig. 5. It is worthwhile to mention that majority of the defects comprised of shrinkage porosity. Although the gas porosity and lack-offusion defects were larger in dimension whenever they were observed, they were relatively sparsely present. Keeping in view the scatter in data, there is negligible difference in defect concentration along the build direction and only a slight difference in defect concentration in different as-built rods, with the overall defect concentration being consistently under $1 \%$.

3.1.3 Phase Constitution. SEM micrographs of the middle regions of the three as-built rods are shown in Fig. 6 and look largely similar. Specimens from the top and bottom of rod 2 , although excluded for the sake of brevity, also exhibited a similar microstructure as shown in Fig. 6. All microstructures are mainly composed of globular precipitates and a bright network-like feature appearing in the SEM (secondary electron, SE) image shown in Fig. 7(a). Such a network has been found to be composed of $\delta$ precipitates (similar in morphology to that shown in Fig. 7(b) but much finer in size) in another published report on EBM built Alloy 718 specimens (Ref 7).

For indexing some of the phases present in the specimens, EDS was employed. Given the fine size of the precipitates, the EDS results shown in Fig. 7 are not very accurate but clearly indicative of the relative elemental distribution present in the various features seen in the micrograph. The phases found in the as-built specimens were $\gamma, \mathrm{MC}$ and $\mathrm{MN}$ as shown in the SEM (SE) image and EDS results for middle region of rod 2 in Fig. 7. The $\delta$ needles $(<1 \mu \mathrm{m})$ [shown in Fig. 7b] precipitating at the grain boundaries were too small to permit reliable EDS analysis. However, the morphology of the precipitates conforms well with other reported studies that have also 


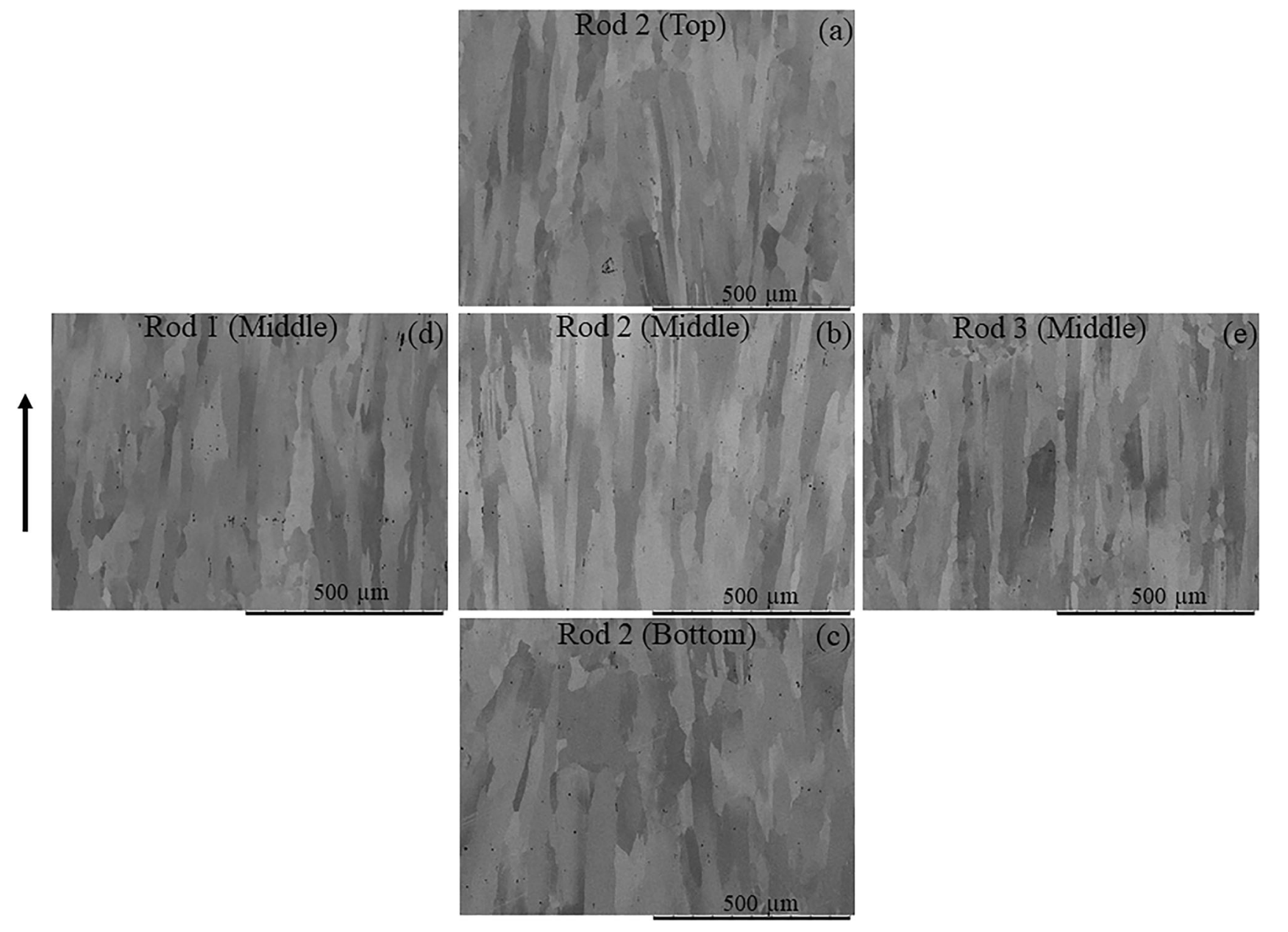

Fig. 3 SEM (BSE) micrographs showing uniform columnar grain structure both within a rod (rod 2) in the build direction and across rods within the build (rod 1-3). Arrow indicates the build direction
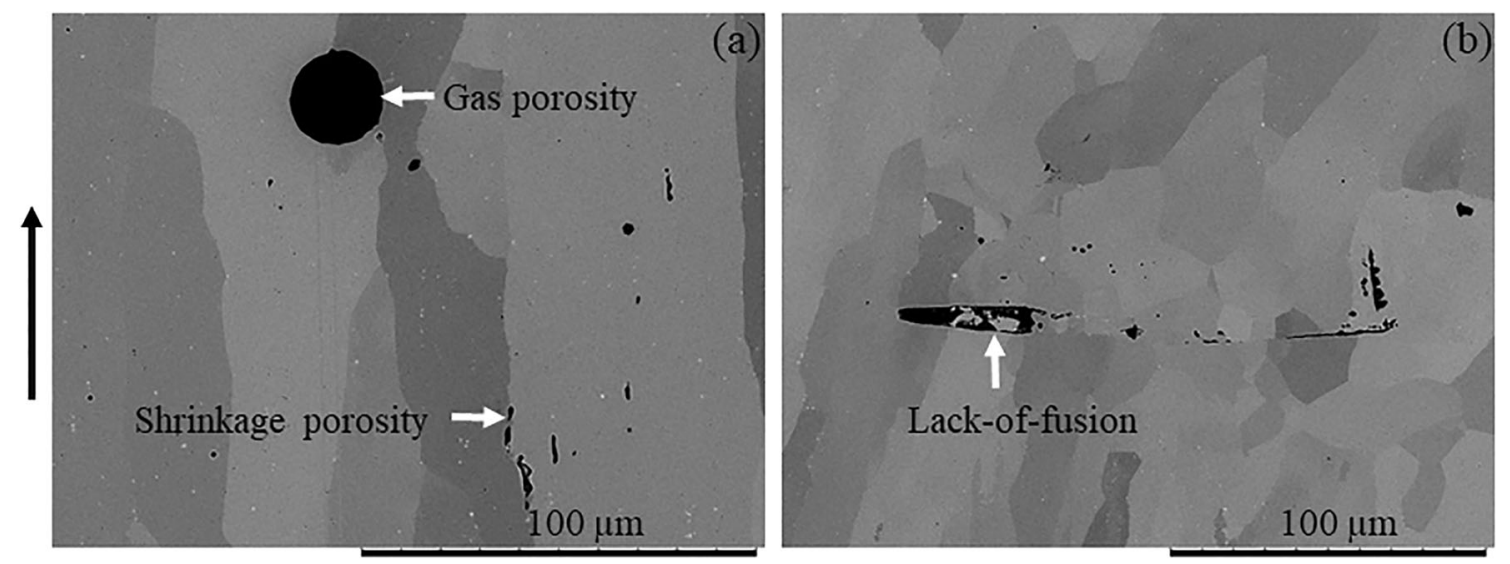

Fig. 4 SEM (BSE) micrographs showing defects in EBM built Alloy 718: (a) gas and shrinkage porosity, (b) lack-of-fusion defect. The black arrow indicates the build direction

identified presence of $\delta$ phase in EBM-built Alloy 718 (Ref 7). $\mathrm{A}$ few $\mathrm{Al}$ and $\mathrm{O}$ rich precipitates were also observed in the microstructure. MC carbides were found to be rich in $\mathrm{Nb}$ and, hence, presumed to be predominantly $\mathrm{NbC}$. Similarly, the MN type precipitates were significantly $\mathrm{Ti}$ rich and were plausibly comprised of TiN precipitates. Similar presence of $\mathrm{NbC}$ and TiN has also been reported in previously published work (Ref
9, 13). It has been previously reported that TiN precipitates act as nucleation sites for $\mathrm{NbC}$ by virtue of the two being isomorphous, (Ref 14) which could plausibly explain the appearance of a dark core in the SEM images of $\mathrm{NbC}$ precipitates, visualized at spot 2 in Fig. 7(a), and the corresponding substantial presence of both $\mathrm{Nb}$ and Ti being detected by $\mathrm{EDS}$. The $\mathrm{NbC}$ phase content was quantified and is 


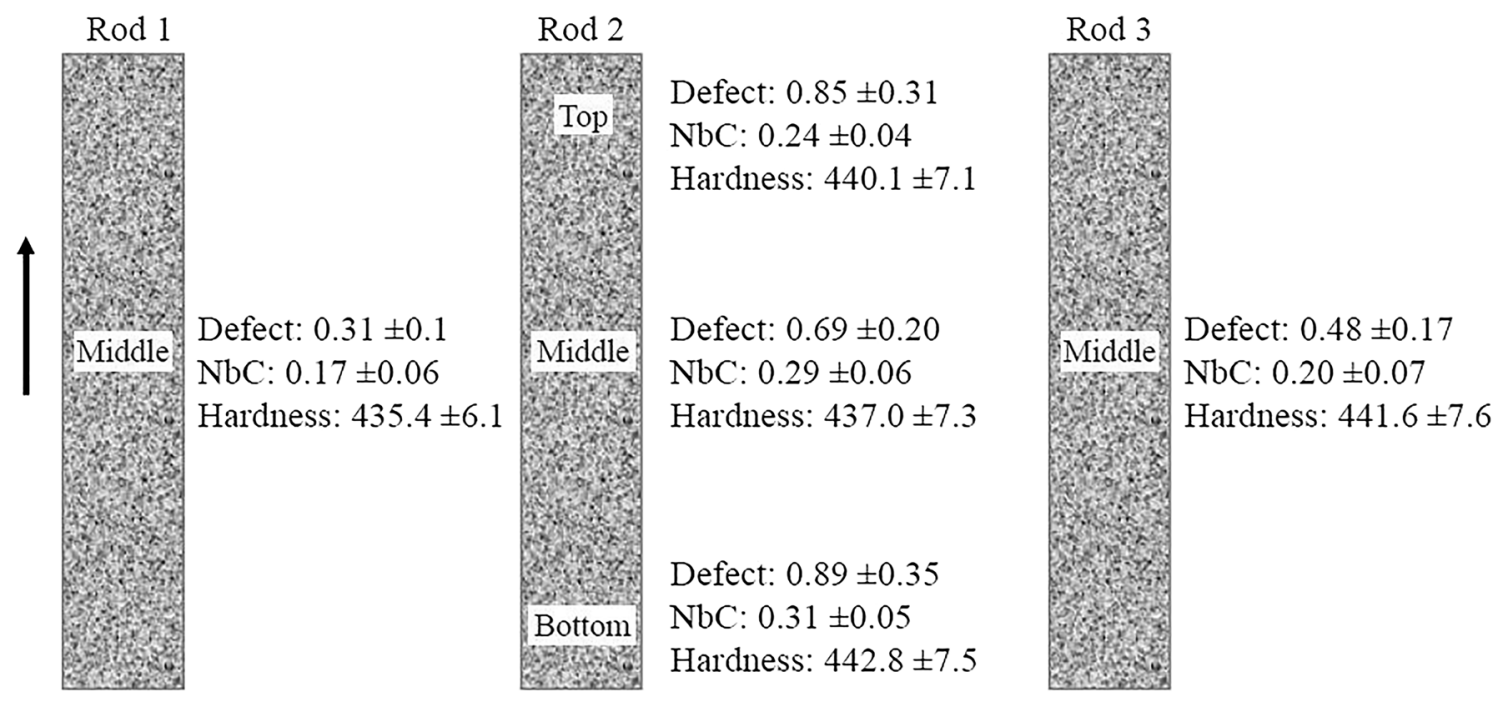

Fig. 5 Schematic of the three as-built rods indicating defects (vol.\%), NbC content (vol.\%), and micro-hardness $\left(\mathrm{HV}_{0.5}\right)$ at different regions of interest. Arrow indicates the build direction
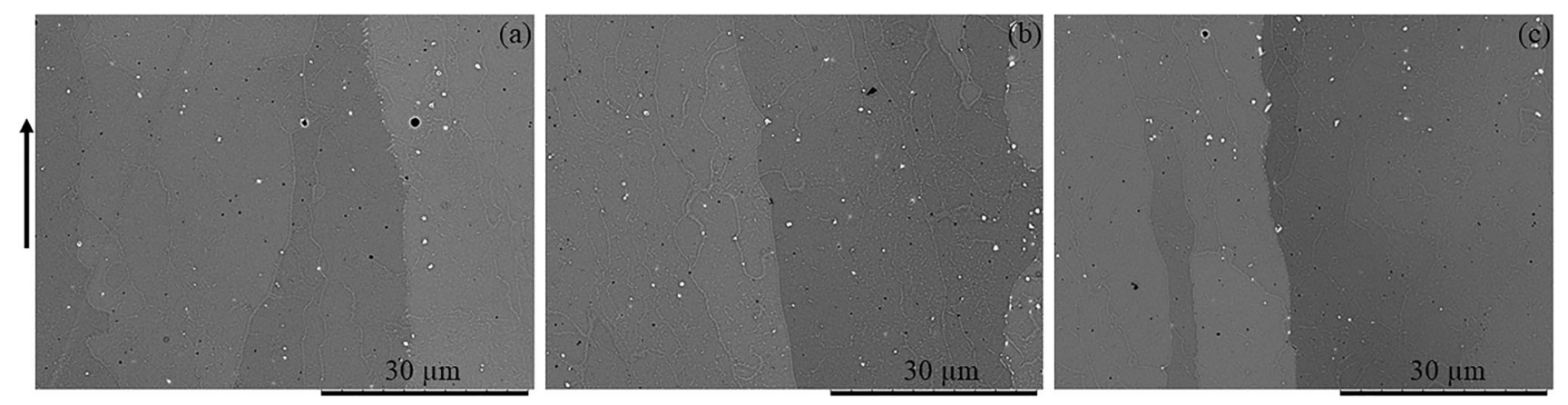

Fig. 6 SEM (BSE) micrograph showing microstructures of specimens from the middle regions of rods (a) 1, (b) 2, and (c) 3 . Arrow indicates the build direction

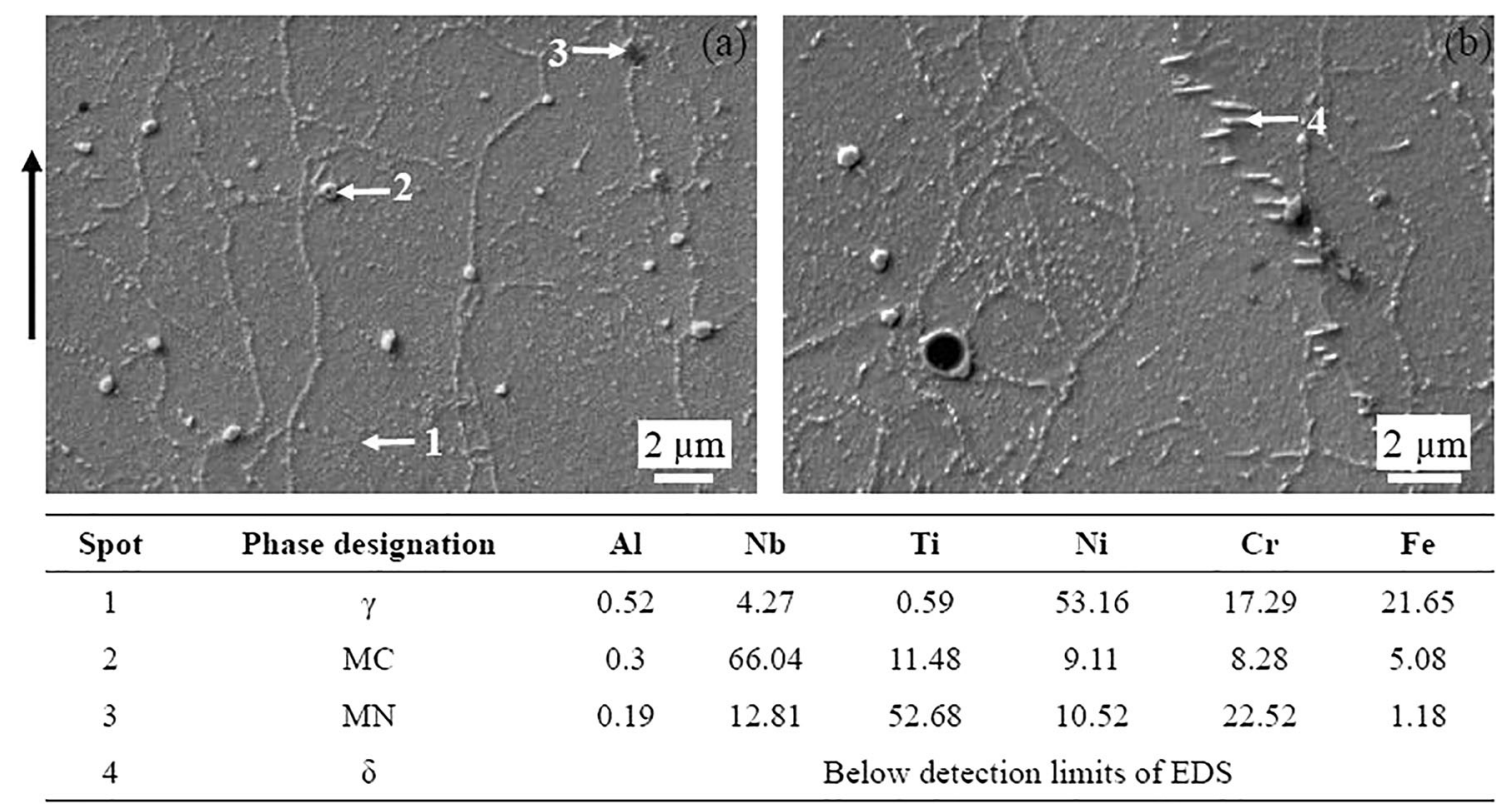

Fig. 7 SEM (SE) micrographs and EDS results for specimen from the middle region of rod 2. The results are given in wt.\%. Arrow indicates the build direction 
summarized in Fig. 5. It can be seen that no statistically significant difference was found between any of the as-built specimens. The presence of $\gamma^{\prime \prime}$ phase has been previously reported in EBM-built Alloy 718, (Ref 7) but it was difficult to visualize it without resorting to high resolution microscopy. Previously published work by the authors had indeed revealed presence of $\gamma^{\prime \prime}$ in the as-built material (Ref 15).

3.1.4 Micro-hardness. The micro-hardness values for all the investigated as-built specimens are given in Fig. 5. Two observations can be made from the hardness results: there is no significant variation in micro-hardness along the build direction or between different rods, and the micro-hardness values of asbuilt specimens exceed the AMS 5662 requirement (350 HV). The high hardness suggests that significant amount of $\gamma^{\prime \prime}$ had precipitated during the EBM process (Ref 9) and was present more or less uniformly throughout the build height, since $\gamma^{\prime \prime}$ phase is the main hardness determinant of Alloy 718 (Ref 16). All the above results amply suggest that there was microstructural uniformity within a rod as well as between different rods in the build.

\subsection{Influence of Post-treatment}

Since microstructural uniformity within a built rod and between different as-built rods has already been established, only middle regions of the rods were taken into consideration for evaluating the influence of post-treatment using the as-built microstructures as the basis.
3.2.1 Grain Structure. A comparison of SEM micrographs of as-built, HIPed, and HIP + HTed specimens shown in Fig. 8 reveals that the columnar grain structure is retained even after the two post treatments. However, there is an increase in the width of the grains after post treatment, it being significantly higher after HIP than after HIP + HT. It could be due to the longer $4 \mathrm{~h}$ exposure to high temperature in case of HIP compared to $3 \mathrm{~h}$ in case of HIP + HT. The increase in grain width in both cases could also be attributed to the dissolution of $\delta$-phase (shown in "Phase Constitution" section) which is reportedly responsible for grain size control in Alloy 718 (Ref 17).

3.2.2 Defects. Figure 9(a) shows the defect content in the post-treated and as-built specimens. It can be seen that the defect content is reduced by more than an order of magnitude in case of both HIP or HIP + HT. Different mechanisms of defect closure during HIPing have been previously suggested and these include plastic deformation, creep, diffusion, etc. (Ref 18, 19). Such extensive reduction of defect content is expected to lead to improvement in mechanical performance of the parts. Previous reports on HIPing of EBM built Alloy 718 have shown significant improvement in low-cycle fatigue of components, with one of the plausible reasons being the healing of defects after HIP (Ref 1).

3.2.3 Phase Constitution. The discernible particulate phases found in case of the as-built specimens discussed earlier and identified to be mainly $\mathrm{NbC}$ with few TiN (Fig. 6 and 7)

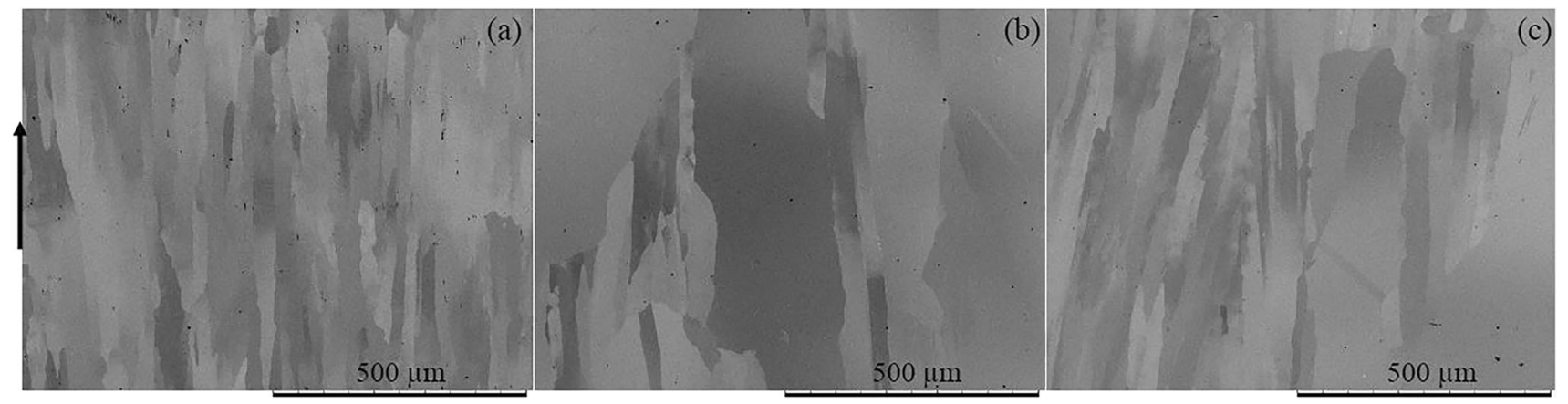

Fig. 8 SEM (BSE) micrographs revealing grain structure of (a) as-built, (b) HIP, and (c) HIP + HT specimens. Arrow indicates the build direction
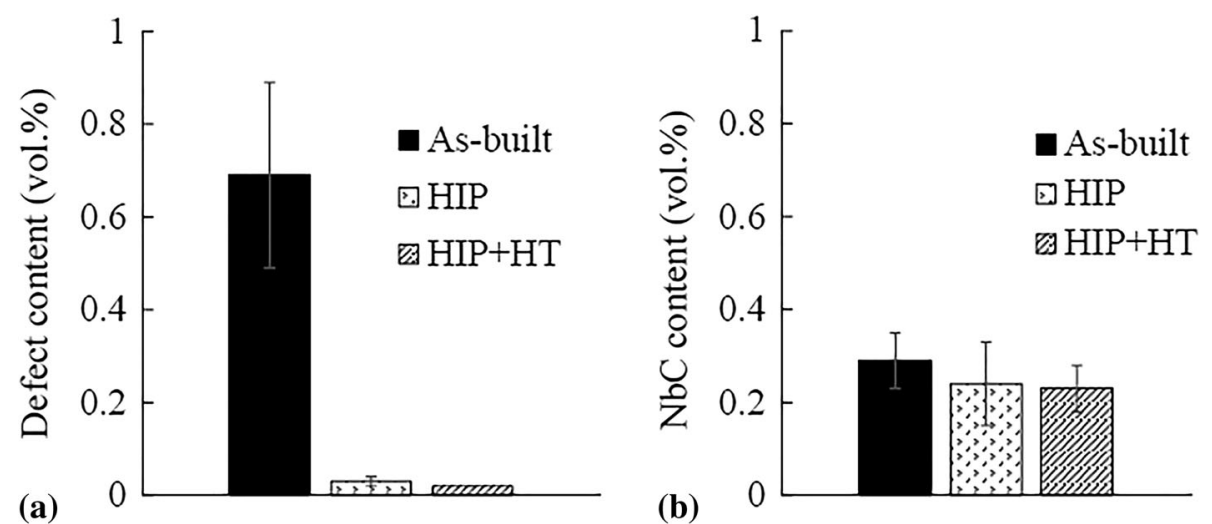

Fig. 9 (a) Defect and (b) NbC content in the middle region of the as-built, HIP, and HIP + HT rods 


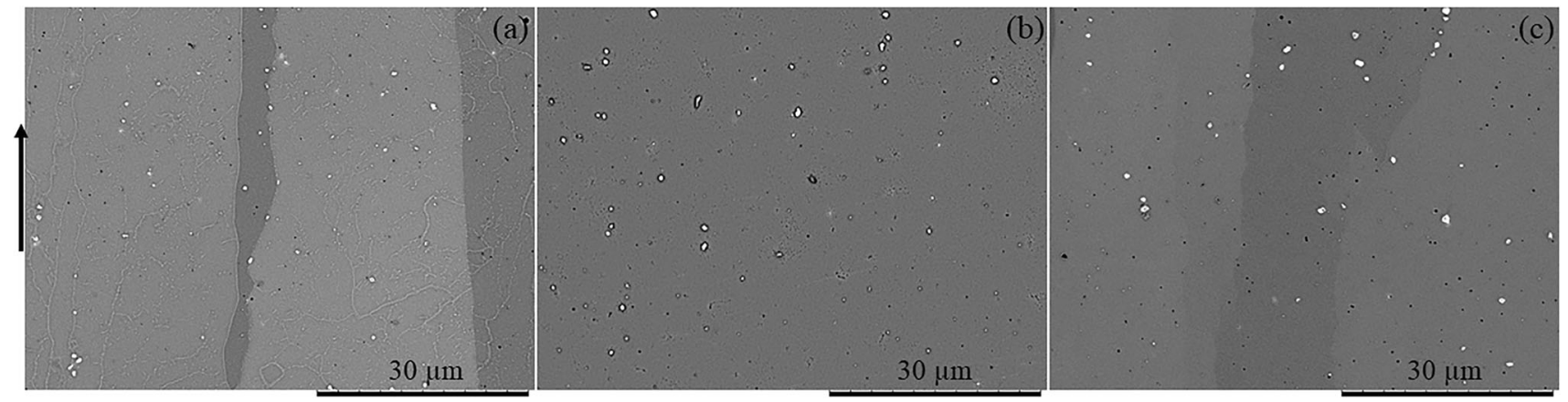

Fig. 10 SEM (BSE) micrographs of specimens in (a) as-built, (b) HIP, and (c) HIP + HT conditions. Arrow indicates the build direction
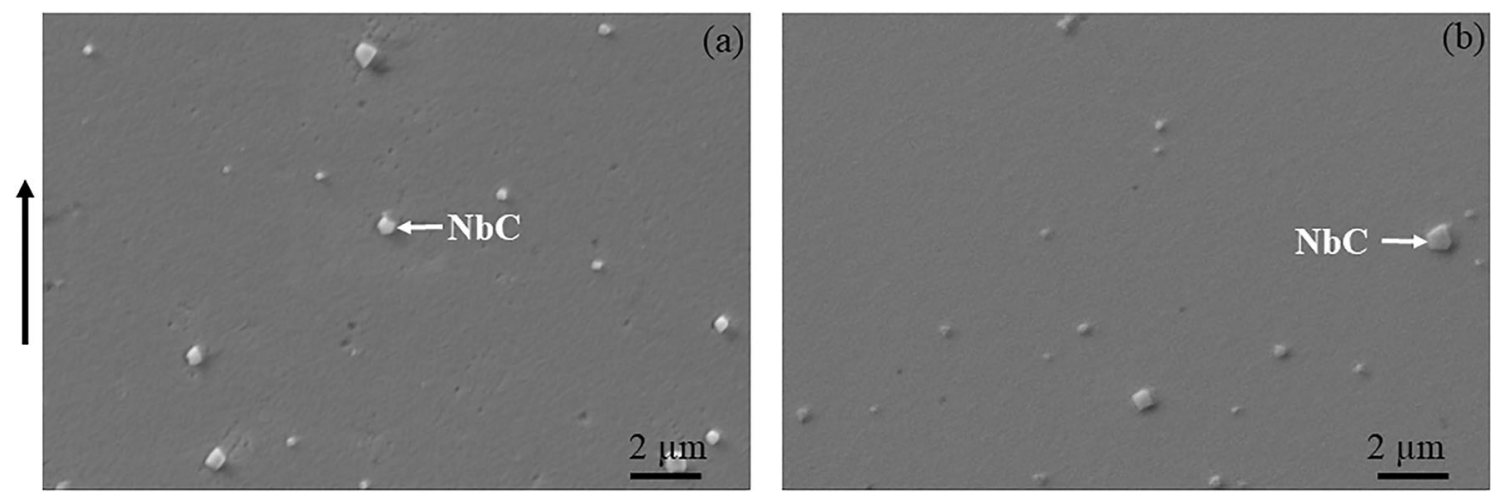

Fig. 11 SEM (SE) micrographs at high magnification showing absence of $\delta$ phase in (a) HIP and (b) HIP + HT condition. Arrow indicates the build direction

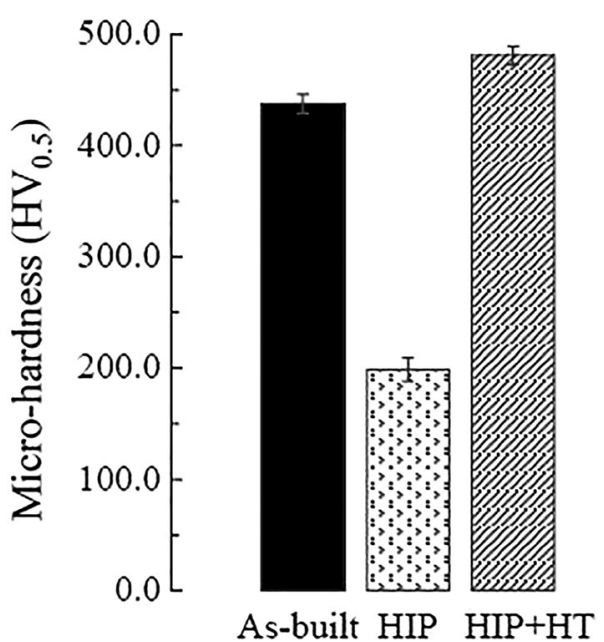

Fig. 12 Micro-hardness of the middle region of the as-built, HIP, and HIP + HT rods

were also found in the HIP and HIP + HT specimens as shown in the SEM micrographs in Fig. 10(b) and (c), respectively. However, no significant difference in the carbide content was noted due to the post-treatment as summarized in Fig. 9(b). The HIP treatment employed was also found to lead to complete dissolution of $\delta$ phase, as can be seen from Fig. 11(a) when compared with Fig. 7 , since the $1200{ }^{\circ} \mathrm{C}$ HIP temperature is above the solvus temperature of $\delta$, which is reportedly $\sim 1010^{\circ} \mathrm{C} \quad(\operatorname{Ref} 17)$. No re-precipitation of $\delta$ was observed during HIP + HT, refer Fig. 11(b), which is in accordance with the reported TTT diagram of Alloy 718 (Ref 20).

3.2.4 Micro-hardness. Significant drop in hardness was observed after HIP treatment as shown in Fig. 12. This could be attributed to the dissolution of $\gamma^{\prime \prime}$ phase, which is the main strengthening phase in Alloy 718. Also, the rapid cooling in the HIP vessel would have inhibited re-precipitation of $\gamma^{\prime \prime}$ in contrast to $\gamma^{\prime \prime}$ precipitation during cooling reported in another study involving slow cooling in HIP vessel (Ref 21). Previous reported work by authors had also shown nearly complete dissolution of $\gamma^{\prime \prime}$ after HIP treatment (Ref 15). Complete dissolution of $\gamma^{\prime \prime}$ after solution treatment at $1050{ }^{\circ} \mathrm{C}$ for $10 \mathrm{~min}$. in case of wrought Alloy 718 has also been reported (Ref 22). HIP + HT led to 'recovery' of hardness and its increase beyond that in the original as-built condition. This can plausibly be attributed to the increased $\gamma^{\prime \prime}$ precipitation resulting from rise in $\mathrm{Nb}$ available in the matrix prior to the aging step, as some $\mathrm{Nb}$ is freed due to dissolution of $\delta$ phase during the HIP step (at $1185^{\circ} \mathrm{C}$ ) of the treatment.

\section{Conclusion}

Based on the results discussed above, the following conclusions can be drawn: 
1. The properties of EBM-built Alloy 718 were found to be uniform, in terms of grain structure, grain width, defect and $\mathrm{NbC}$ content, and hardness, along the build height. Similar uniformity was also found to exist among specimens built at different locations on the base plate.

2. HIP led to reduction in the defect content of the material by more than an order of magnitude.

3. The HIP temperature used in the present study led to nearly complete dissolution of the $\delta$ and $\gamma^{\prime \prime}$ phase, and was accompanied by significant reduction in hardness.

4. HIP + HT treatment led to sharp 'recovery' of hardness which can be attributed to precipitation of $\gamma^{\prime \prime}$ phase.

\section{Acknowledgments}

Kenneth Andersson is acknowledged for help and guidance during SEM and EDS characterization. Financial assistance by the Knowledge Foundation (20160281) is gratefully acknowledged.

\section{Open Access}

This article is distributed under the terms of the Creative Commons Attribution 4.0 International License (http://creativecommons.org/ licenses/by/4.0/), which permits unrestricted use, distribution, and reproduction in any medium, provided you give appropriate credit to the original author(s) and the source, provide a link to the Creative Commons license, and indicate if changes were made.

\section{References}

1. M.M. Kirka, D.A. Greeley, C. Hawkins, and R.R. Dehoff, Effect of Anisotropy and Texture on the Low Cycle Fatigue Behavior of Inconel 718 Processed Via Electron Beam Melting, Int. J. Fatigue, 2017, 105, p 235-243

2. M.M. Kirka, F. Medina, R. Dehoff, and A. Okello, Mechanical Behavior of Post-Processed Inconel 718 Manufactured Through the Electron Beam Melting Process, Mater. Sci. Eng. A, 2016, 680, p 1-9

3. D. Deng, J. Saarimäki, H. Söderberg, R. L. Peng, H. Brodin, and J. Moverare, Microstructural Characterization of As-Manufactured and Heat Treated Electron Beam Melted Inconel 718, Materials Science and Technology, Oct 23-27, 2016 (Salt Lake City), MS\&T, 2016, p $105-112$

4. S.-H. Chang, S.-C. Lee, T.-P. Tang, and H.-H. Ho, Influences of Soaking Time in Hot Isostatic Pressing on Strength of Inconel 718 Superalloy, Mater. Trans., 2006, 47, p 426-432

5. W. Tillmann, C. Schaak, J. Nellesen, M. Schaper, M.E. Aydinöz, and K.-P. Hoyer, Hot Isostatic Pressing of IN718 Components Manufactured by Selective Laser Melting, Addit. Manuf., 2017, 13, p 93-102

6. Standard Practice for determining the Inclusion or Second-Phase Constituent Content of Metals by Automatic Image Analysis. E124503, ASTM-International, 2008, p 1-8
7. M.M. Kirka, K.A. Unocic, N. Raghavan, F. Medina, R.R. Dehoff, and S.S. Babu, Microstructure Development in Electron Beam-Melted Inconel 718 and Associated Tensile Properties, JOM, 2016, 68, p 1012-1020

8. M.M. Kirka, Y. Lee, D.A. Greeley, A. Okello, M.J. Goin, M.T. Pearce, and R.R. Dehoff, Strategy for Texture Management in Metals Additive Manufacturing, JOM, 2017, 69, p 523-531

9. D. Deng, J. Moverare, R.L. Peng, and H. Söderberg, Microstructure and Anisotropic Mechanical Properties of EBM manufactured Inconel 718 and Effects of Post Heat Treatments, Mater. Sci. Eng. A, 2017, 693, p 151-163

10. S.P. Narra, R. Cunningham, J. Beuth, and A.D. Rollett, Location Specific Solidification Microstructure Control in Electron Beam Melting of Ti-6Al-4V, Addit. Manuf., 2018, 19, p 160-166

11. X. Wang, T. Keya, and K. Chou, Build Height Effect on the Inconel 718 Parts Fabricated by Selective Laser Melting, Procedia Manuf., 2016, 5, p 1006-1017

12. W.J. Sames, F.A. List, S. Pannala, R.R. Dehoff, and S.S. Babu, The Metallurgy and Processing Science of Metal Additive Manufacturing, Int. Mater. Rev., 2016, 61, p 315-360

13. S. L. Cockcroft, T. Degawa, A. Mitchell, D. W. Tripp, and A. Schmalz, Inclusion precipitation in superalloys, in Superalloys, S. D. Antolovich, R. W. Stusrud, R. A. MacKay, D. L. Anton, T. Khan, R. D. Kissinger, D. L. Klarstrom, Ed., Sept 20-24, 1992 (Champion), The Minerals, Metals \& Materials Society, 1992, p 577-586

14. A. Mitchell, Primary Carbides in Alloy 718, in Superalloy 718 and Derivatives, E.A. Ott, J.R. Groh, A. Banik, I. Dempster, T.P. Gabb, R. Helmink, X. Liu, A. Mitchell, G.P. Sjöberg, and A. WusatowskaSarnek, Ed., Oct 10-13, 2010 (Pittsburgh), Wiley-TMS, 2010, p 161167

15. S. Goel, J. Olsson, M. Ahlfors, U. Klement, and S. Joshi, The Effect of Location and Post-treatment on the Microstructure of EBM-Built Alloy 718, in Superalloy 718 and Derivatives, E. Ott, X. Liu, J. Andersson, Z. Bi, K. Bockenstedt, I. Dempster, J. Groh, K. Heck, P. Jablonski, M. Kaplan, D. Nagahama, and C. Sudbrack, Ed., June 3-6, 2018 (Pittsburgh), Springer, 2018, p 115-131

16. M. Anderson, A.-L. Thielin, F. Bridier, P. Bocher, and J. Savoie, $\delta$ Phase Precipitation in Inconel 718 and Associated Mechanical Properties, Mater. Sci. Eng. A, 2017, 679, p 48-55

17. J.F. Radavich, The Physical Metallurgy of Cast and Wrought Alloy 718, in Superalloy 718-Metallurgy and Applications, E.A. Loria, Ed., June 12-14, 1989 (Pittsburgh), Minerals, Metals \& Materials Society, 1989, p 229-240

18. J. Li, C. Yuan, J. Guo, J. Hou, and L. Zhou, Effect of Hot Isostatic Pressing on Microstructure of Cast Gas-Turbine Vanes of K452 Alloy, Int. Prog. Nat. Sci. Mater., 2014, 24, p 631-636

19. P.G. Bailey and W.H. Schweikert, HIP Densification of Castings, in Superalloys, B.H. Kear, Ed., Sept 12-15, 1976 (Seven Springs), Claitor's Pub. Division, 1976, p 451-462

20. J.W. Brooks and P.J. Bridges, Metallurgical Stability of Inconel 718, in Superalloy, S. Reichman, D.N. Duhl, G. Maurer, S. Antolovich, and C. Lun, Ed., Sept 18-22, 1988 (Champion), Miner. Metals Mater. Soc., 1988, pp. 33-42

21. K.A. Unocic, L.M. Kolbus, R.R. Dehoff, S.N. Dryepondt, and B.A. Pint, High-Temperature Performance of UNS N07718 Processed by Additive Manufacturing, in Corrosion, Mar 9-13, 2014 (San Antonio), NACE International, 2014

22. L.C.M. Valle, L.S. Araújo, S.B. Gabriel, J. Dille, and L.H. de Almeida, The Effect of $\delta$ Phase on the Mechanical Properties of an Inconel 718 Superalloy, J. Mater. Eng. Perform., 2013, 22, p $1512-1518$ 